\title{
Evaluación Ultrasonográfica del Tejido Blando Facial en Adultos Chilenos
}

\author{
Ultrasonic Assessment of Facial Soft Tissue in Adult Chileans
}

Iván Claudio Suazo Galdames; Guillermo Enrique Salgado Alarcón \& Mario Gonzalo Cantín López

\begin{abstract}
SUAZO, G. I. C.; SALGADO, A. G. E.; CANTÍN, L. M. G. Evaluación ultrasonográfica del tejido blando facial en adultos chilenos. Int. J. Morphol., 25(3):643-648, 2007.

RESUMEN: Existen variadas técnicas para realizar la identificación médicolegal en restos cadavéricos u osamentas. Una de ellas es la identificación por escultura forense. Para esto, se necesitan ciertas referencias en la relación entre tejido óseo y tejido blando. El objetivo de esta investigación fue determinar los grosores tisulares faciales mediante ultrasonografía y correlacionarlos con edad y sexo. Se seleccionó una muestra de 48 personas, entre 21 y 50 años de edad de ambos sexos, que consultaron al Servicio de Imagenología de la Clínica Multimédica de Talca, Chile, distribuidas en dos grupos: A: 12 hombres y 12 mujeres entre 21 y 35 años de edad, B: 12 hombres y 12 mujeres entre 36 y 50 años de edad. Para este estudio se utilizó un ecógrafo General Electric ®, modelo Voluson 730 expert, con el cual se midieron los grosores tistulares en 15 puntos medianos y paramedianos de la cabeza. Los resultados de la medición de los grosores titulares mostraron diferencias significativas entre grupos etáreos y sexos. No se observaron diferencias significativas, entre las personas del mismo sexo de un mismo grupo etáreo.
\end{abstract}

PALABRAS CLAVE: Forense; Reconstitución facial; Grosor de tejido blando; Identificación.

\section{INTRODUCCIÓN}

En identificación Médico Legal, identificación es la acción y efecto de identificar, esto es, constatar la individualidad de la persona humana, reconocer y comprobar que es la misma que se supone o busca (Teke, 2004).

Cuando la identificación es en restos cadavéricos u osamentas, los métodos como huellas dactilares, documentos, reconocimiento ocular por testigos, entre otros, no sirven para lograr saber de quién se trata, por lo tanto, se debe recurrir a otras técnicas.

La reconstrucción facial es una técnica odontoantropológica cuyo objetivo es realizar una estimación de la cara de un individuo a la hora de la muerte, sobre el macizo u osamenta craneofacial, basado en ciertos puntos craneométricos y en el espesor de los tejidos blandos (Wilkinson, 2002; Claes et al., 2006; Utsuno et al., 2007).

El principal fundamento de la reconstrucción facial forense es que las proporciones del tejido óseo definen la forma facial, indicándose que el cráneo es la matriz de la cabeza viva, siendo la base de los tejidos de la cabeza y de la cara (Wilkinson et al., 2003). Diversos autores han estudiado las correlaciones existentes entre los tejidos óseo y blando, en distintos puntos de la cabeza que corresponden a puntos cefalométricos constantes y claramente identificables, que sirven de guía para la reconstitución facial por escultura forense (Bergman, 1999; Utsuno et al., 2007; Claes et al.). Smith \& Buschang (2001) observaron esta relación entre tejidos óseo y blando, concluyendo que la forma facial va a depender de las proporciones del tejido óseo.

Utsuno et al. (2007) plantean que es necesaria mayor información para reconstruir el rostro, tales como color del pelo, forma de los párpados, perfil de la nariz, oreja, etc. y la mayor parte de estas características se han perdido o dañado al momento de encontrar el cráneo (Vandermeulen et al., 2006).

Los protocolos de reconstrucción craneofacial requieren la identificación de aspectos como raza, sexo y edad para el correcto desarrollo de la técnica de reconstrucción craneofacial para, de esta manera, poder seleccionar correctamente las profundidades de los tejidos (Domaracki \& Stephan, 2006) 
Los espesores de los tejidos faciales son datos imprescindibles para la reconstrucción facial, porque todas las técnicas de reconstrucción facial se basan en el espesor de los tejidos blandos (Utsuno et al., 2007).

Stephan \& Henneberg (2001) realizaron una evaluación de varios métodos de reconstrucción facial ocupando cadáveres para la obtención de datos y concluyeron que no era posible reproducir al individuo en forma fidedigna, ya que la identificación por parte de familiares no fue más de un $38 \%$. El problema principal de trabajar con cadáveres para determinar grosores, es el fenómeno físico de la deshidratación de los tejidos blandos (10-18 gramos/día/peso), además del rigor mortis que afecta a las fibras musculares (De Greef et al., 2006).

La medición de grosores tisulares con exploración tridimensional, ya sea con radiación, ultrasonido o un programa computacional, entrega datos fidedignos para la reconstrucción facial, siendo ésta más exacta (De Greef \& Willems, 2005; De Greef et al.), ya que elimina los factores nombrados anteriormente, aunque la recolección de datos con estos métodos sea más lento. (Utsuno et al., 2005).

El-Mehallawi \& Soliman (2001) plantean que en el espesor de los tejidos blandos faciales adultos existe un marcado dimorfismo sexual, siendo mayor el espesor en el sexo femenino que en el masculino, especialmente en las regiones del párpado (punto supraorbital), boca, labios, mentón y mandíbula (punto gonion); resultados similares a los comunicados por Suazo et al. (2007), quienes describieron un mayor grosor en los tejidos blandos, en puntos paramedianos de la cara en mujeres, mientras que en hombres éstos fueron mayores en relación a los puntos medianos observados. Estos espesores no tienen variaciones estadísticamente significativas entre un lado y otro de la cara de un mismo individuo (De Greef et al., Suazo et al.).

Utsuno et al. (2005) plantean que los espesores del tejido blando facial no tienen diferencias estadísticamente significativas por sexo en menores de 11 años, pero después de esta edad las diferencias por sexo comienzan a aparecer y aumentan en las regiones superior e inferior de la cara. También estos autores observan que los puntos en la zona mediana de la cara, en el sexo femenino, disminuyen a medida que se avanza en edad.

En nuestra investigación se realizó la determinación de grosores tisulares en distintos puntos medianos y paramedianos de la cara en sujetos adultos de la región del Maule, ocupando el ultrasonido como instrumento de medición por las propiedades señaladas anteriormente, con el fin de contribuir al conocimiento de las profundidades del tejido blando facial en población investigada.

\section{MATERIAL Y MÉTODO}

En este estudio participaron 48 personas con edades entre 21 y 50 años (media 34.4 años, D.S. 8.6), relación pesotalla normal (índice de masa corporal), que acudieron al Servicio de Imagenología de la Clínica Multimédica de Talca. Luego de ser informadas de la naturaleza del estudio, accedieron voluntariamente a participar, dejando constancia escrita de su aceptación.

No se consideraron para este estudio los pacientes con deformidades o cicatrices faciales y los que habían sido sometidos a procedimientos de cirugía plástica facial u ortognática.

La muestra fue dividida en dos grupos de acuerdo a edad:

Grupo A: 12 mujeres y 12 hombres entre 21 y 35 años (media 26.9 años, D.S. 4.1).

Grupo B: 12 mujeres y 12 hombres entre 36 y 50 años (media 41.9 años, D.S. 4.2).

En cada paciente se identificaron en total 15 puntos céfalo-métricos, 8 de ellos medianos: trichion, supraglabela, glabela, nasion, A' de downs, B' de downs, pogonion y gnathion; $\mathrm{y} 7$ puntos paramedianos: superciliar, supraorbiatrio, infraorbitario, lateral de la orbita (exocanthion), zygion, agujero mentoniano y gonion, los cuales fueron marcados con un lápiz dermográfico. En la Fig. 1 se presentan los puntos medianos y paramedianos marcados.

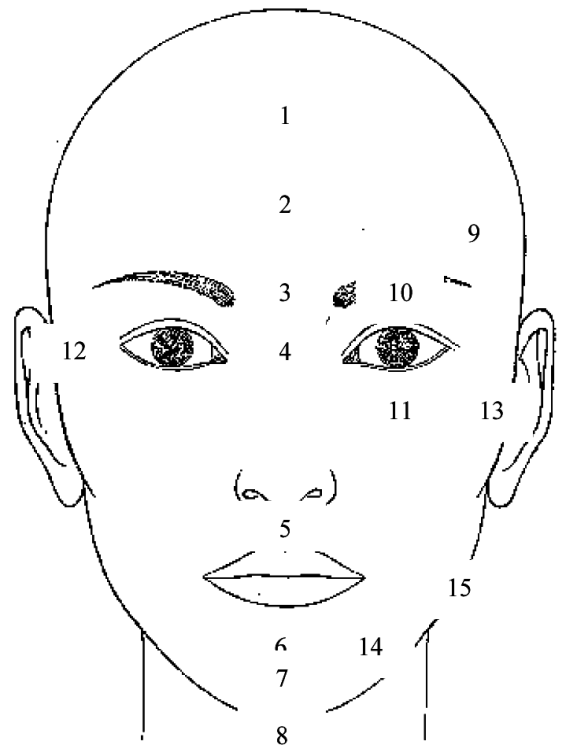

Fig. 1. Puntos faciales medianos: 1 Trichion; 2. Supraglabela; 3. Glabela; 4. Nasion; 5. A' de downs; 6. B' de downs; 7. Pogonion; 8. Gnathion; y paramedianos: 9. Superciliar; 10. Supraorbiatrio; 11. Infraorbitario; 12. Exocanthion; 13. Zygion; 14. Agujero mentoniano; 15. Gonion. 
En cada punto marcado se aplicó gel, y se ubicó el transductor (mod. ATL L12-5 50mm Ultrasound Transluce, General Electric $\left.{ }^{\circledR}\right)$ del ecógrafo general electric mod. Voluson 730 expert. Una vez localizado el punto en la pantalla, se congeló la imagen y se midió la distancia, en milímetros, mediante una línea recta perpendicular al plano profundo que correspondería a reparo óseo.

La determinación de la ubicación de los puntos y el uso del ecógrafo la realizó siempre el mismo operador.

Se registraron los valores de los puntos a medir en una ficha con los datos correspondientes al paciente: nombre, edad, sexo, peso e índice de masa corporal, los cuales fueron tabulados y analizadas sus correlaciones mediante el test de Tukey.

\section{RESULTADOS}

Para los hombres del grupo A, se observaron los siguientes resultados promedio en milímetros en cada punto cefalométrico: Triquion $=4,966$; Supraglabela $=$ 4,983; Glabela $=4,833$; Nasion $=5,683$; A' de downs = 11,$003 ;$ B' $^{\prime}$ de downs $=11,183$; Pogonion $=10,175$; Gnathion $=7,541$; Superciliar $=4,783$; Supraorbiatrio $=$ 6,033; Exocanthion = 5,991; Infraorbitario $=7,166$; Zygion $=6,791 ;$ Agujero mentoniano $=12,791 ;$ Gonion $=17,74$.

Para las mujeres del grupo A, se observaron los siguientes resultados promedio en milímetros en cada punto cefalométrico: Triquion $=4,141$; Supraglabela $=$ 4,458; Glabela $=4,791 ;$ Nasion $=5,191 ; \mathrm{A}^{\prime}$ de downs $=$ 8,791; B' de downs = 8,533; Pogonion $=9,191$; Gnathion $=5,791 ;$ Superciliar $=5,275 ;$ Supraorbiatrio $=5,983$; Exocanthion $=4,625 ;$ Infraorbitario $=5,025 ;$; Zygion $=$ 9,9; Agujero mentoniano $=11,025 ;$ Gonion $=14,81$.

Para los hombres del grupo B, se observaron los siguientes resultados promedio en milímetros en cada punto cefalométrico: Triquion $=5,05 ;$ Supraglabela $=5,2$; Glabela $=5,091 ;$ Nasion $=4,775 ; A^{\prime}$ de downs $=10,191$; B' de downs =9,616; Pogonion =9,016; Gnathion =6,116; Superciliar $=5,1 ;$ Supraorbiatrio $=5,775 ;$ Exocanthion $=$ 4,441; Infraorbitario $=6,783 ;$ Zygion $=8,658$; Agujero mentoniano $=10,841 ;$ Gonion $=14,96$.

Para las mujeres del grupo B, se observaron los siguientes resultados promedio en milímetros en cada punto cefalométrico: Triquion $=4,841$; Supraglabela $=$
5,308; Glabela $=5,866 ;$ Nasion $=5,683 ; \mathrm{A}^{\prime}$ de downs $=$ 10,$208 ; B^{\prime}$ de downs $=9,558 ;$ Pogonion $=10,208$; Gnathion $=8,75 ;$ Superciliar $=7,691$; Supraorbiatrio $=$ 5,508; Exocanthion $=5,558$; Infraorbitario $=7,083$; Zygion $=10,633 ;$ Agujero mentoniano $=10,65 ;$ Gonion $=11,516$.

Los grosores promedio de cada punto por grupo, la desviación típica, el error típico, el mínimo y el máximo se encuentran resumidos en Tabla I.

$\mathrm{Al}$ correlacionar los datos obtenidos entre hombres y mujeres del grupo A, se puede observar que todos los puntos cefalométricos en los hombres presentan un mayor grosor que las mujeres, a excepción del punto zygion.

El mismo análisis de datos, entre hombres y mujeres del grupo B, muestra que los hombres presentan menor grosor tisular en la mayoría de los puntos cefalométricos, pero en cuatro de ellos el grosor es mayor, correspondiendo a los puntos triquion, B' de downs, supraorbitario y gonion.

$\mathrm{Al}$ analizar individuos del mismo sexo pero de distinto grupo etáreo, se observa que los hombres del grupo A presentan mayor grosor tisular que los hombres del grupo B; sin embargo, cinco puntos cefalométricos presentaron menor grosor, siendo éstos: triquion, supraglabela, glabela, superciliar y zygion.

De la misma forma, las mujeres del grupo A presentan menor grosor tisular que las mujeres del grupo B en los puntos cefalométricos investigados, a excepción de tres puntos donde el grosor resulta ser mayor, siendo éstos: supraorbitario, agujero mentoniano y gonion.

\section{DISCUSIÓN}

De acuerdo a los resultados se puede concluir que existen diferencias morfológicas estadísticamente significativas entre individuos de distinto sexo y de igual grupo etáreo, y entre individuos del mismo sexo, pero de distinto grupo etáreo.

Estos resultados concuerdan con Stephan et al. (2005) quienes justifican el estudio tanatológico del cráneo antes de una reconstrucción, por el dimorfismo sexual que presentan los valores de grosor tisular.

También se observaron diferencias atribuibles a la edad. En este estudio se obtuvieron diferencias significa- 
Tabla I. Datos estadísticos de grosores tisulares por sexo y grupo.

\begin{tabular}{|c|c|c|c|c|c|c|c|c|c|c|c|c|c|c|c|}
\hline \multicolumn{16}{|c|}{ HOMBRES GRUPO A } \\
\hline & tr & $\mathrm{sg}$ & $\mathrm{g}$ & $\mathrm{n}$ & $\begin{array}{l}\mathrm{A}^{\prime} \mathrm{de} \\
\text { Downs }\end{array}$ & $\begin{array}{c}\text { B' de } \\
\text { Downs }\end{array}$ & $\mathrm{pg}$ & gn & sci & so & ex & io & $\mathrm{zy}$ & $\begin{array}{l}\text { Aguj. } \\
\text { ment. }\end{array}$ & go \\
\hline Promedio & 4,966 & 4,983 & 4,833 & 5,683 & 11,003 & 11,183 & 10,175 & 7,541 & 4,783 & 6,033 & 5,991 & 7,166 & 6,791 & 12,791 & 17,74 \\
\hline Des. típica & ,2498 & ,298 & ,4334 & ,2918 & ,4033 &, 4840 &, 4731 & ,3579 & ,2725 &, 3143 & ,2999 &, 3312 & ,3728 &, 2466 & ,3679 \\
\hline Err. típico &, 1018 & ,1293 &, 1230 &, 1139 & , 1699 &, 1483 &, 1683 &, 1428 &, 1321 & ,1224 &, 116 & , 1409 &, 1423 &, 117 & ,2109 \\
\hline Mínimo & 4,6 & 4,5 & 4,2 & 5,3 & 10,6 & 10,6 & 9,6 & 7 & 4,4 & 5,6 & 5,6 & 6,6 & 6,2 & 12,5 & 17,2 \\
\hline Máximo & 5,3 & 5,4 & 5,7 & 6,1 & 11,8 & 12,2 & 11 & 8,1 & 5,2 & 6,5 & 6,6 & 7,7 & 7,2 & 13,2 & 18,3 \\
\hline \multicolumn{16}{|c|}{ MUJERES GRUPO A } \\
\hline & $\operatorname{tr}$ & $\mathrm{sg}$ & $\mathrm{g}$ & $\mathrm{n}$ & $\begin{array}{l}\mathrm{A}^{\prime} \mathrm{de} \\
\text { Downs }\end{array}$ & $\begin{array}{c}\text { B' de } \\
\text { Downs }\end{array}$ & $\mathrm{pg}$ & gn & sci & so & ex & io & zy & $\begin{array}{l}\text { Aguj. } \\
\text { ment. }\end{array}$ & go \\
\hline Promedio & 4,141 & 4,458 & 4,791 & 5,191 & 8,791 & 8,533 & 9,191 & 5,791 & 4,275 & 5,983 & 4,625 & 5,025 & 9,9 & 11,025 & 14,81 \\
\hline Des. típica &, 2275 &, 337 & ,2234 &, 2275 & ,4358 & ,4030 & ,4188 & ,4078 & ,4003 & ,3010 & ,2667 & ,3306 & ,4112 & ,2667 & ,6708 \\
\hline Err. típico &, 1018 &, 1293 &, 1230 &, 1139 & , 1699 &, 1483 &, 1683 &, 1428 &, 1321 & , 1224 &, 116 & , 1409 &, 1423 &, 117 & ,2109 \\
\hline Mínimo & 3,8 & 3,9 & 4,4 & 4,8 & 8,3 & 7,9 & 8,4 & 5,2 & 3,7 & 5,4 & 4,3 & 4,6 & 9,2 & 10,4 & 14,1 \\
\hline Máximo & 4,5 & 4,9 & 5,1 & 5,5 & 9,5 & 9 & 9,9 & 6,5 & 4,8 & 6,4 & 5,1 & 5,8 & 10,4 & 11,3 & 16 \\
\hline \multicolumn{16}{|c|}{ HOMBRES GRUPO B } \\
\hline & $\operatorname{tr}$ & $\mathrm{sg}$ & $\mathrm{g}$ & $\mathrm{n}$ & $\begin{array}{c}\mathrm{A}^{\prime} \mathrm{de} \\
\text { Downs }\end{array}$ & $\begin{array}{c}\text { B' de } \\
\text { Downs }\end{array}$ & $\mathrm{pg}$ & gn & sci & so & ex & io & zy & $\begin{array}{l}\text { Aguj. } \\
\text { ment. }\end{array}$ & go \\
\hline Promedio & 5,05 & 5,2 & 5,091 & 4,775 & 10,191 & 9,616 & 9,016 & 6,116 & 5,1 & 5,775 & 4,441 & 6,783 & 8,658 & 10,841 & 14,96 \\
\hline Des. típica & ,309 & ,3931 &, 2429 &, 3519 & ,4926 &, 2517 &, 3512 & ,3271 & ,2923 & ,2454 & ,2678 & ,3589 & ,3260 &, 3825 &, 576 \\
\hline Err. típico &, 1018 & , 1293 &, 1230 &, 1139 & , 1699 &, 1483 &, 1683 & , 1428 &, 1321 & , 1224 &, 116 &, 1409 &, 1423 &, 117 & ,2109 \\
\hline Mínimo & 4,5 & 4,7 & 4,7 & 4,3 & 9,3 & 9,3 & 8,5 & 5,6 & 4,7 & 5,4 & 4 & 6 & 8 & 10,5 & 14,2 \\
\hline Máximo & 5,5 & 5,7 & 5,5 & 5,3 & 10,9 & 10 & 9,6 & 6,6 & 5,5 & 6,2 & 4,8 & 7,4 & 9 & 11,9 & 15,9 \\
\hline \multicolumn{16}{|c|}{ MUJERES GRUPO B } \\
\hline & $\operatorname{tr}$ & $\mathrm{sg}$ & $\mathrm{g}$ & $\mathrm{n}$ & $\begin{array}{l}\mathrm{A}^{\prime} \mathrm{de} \\
\text { Downs }\end{array}$ & $\begin{array}{c}\text { B' de } \\
\text { Downs }\end{array}$ & $\mathrm{pg}$ & gn & sci & so & ex & io & zy & $\begin{array}{l}\text { Aguj. } \\
\text { ment. }\end{array}$ & go \\
\hline Promedio & 4,841 & 5,308 & 5,866 & 5,683 & 10,208 & 9,558 & 10,208 & 8,75 & 7,691 & 5,508 & 5,558 & 7,083 & $\begin{array}{c}10,63 \\
3\end{array}$ & 10,650 & $\begin{array}{c}11,51 \\
6\end{array}$ \\
\hline Des. típica & ,2429 & 2109 &, 257 &, 225 & ,3118 & ,261 & ,3965 & 297 &, 3147 & ,3315 & ,2999 & ,3589 & ,2674 & ,2236 & ,3881 \\
\hline Err. típico &, 1018 & ,1293 & , 1230 & ,1139 & ,1699 &, 1483 &, 1683 &, 1428 &, 1321 & , 1224 &, 116 &, 1409 &, 1423 & , 117 & ,2109 \\
\hline Mínimo & 4,5 & 4,9 & 5,4 & 5,4 & 9,7 & 9,2 & 9,7 & 8,4 & 7,2 & 5,1 & 5 & 6,5 & 10,3 & 11,2 & 10,7 \\
\hline Máximo & 5,2 & 5,6 & 6,3 & 6,1 & 10,6 & 10 & 10,7 & 9,2 & 8,2 & 6 & 6 & 7,6 & 11,2 & 12 & 12,1 \\
\hline
\end{tabular}

tivas entre grupos etáreos, lo que coincide con Smith \& Buschang, que indican que factores como la falta de desarrollo cráneo-facial, arrugas faciales, flacidez de los tejidos, factores físicos como la gravedad y deshidratación afectan proporcionalmente al tejido blando y, por consiguiente, el grosor tisular, de acuerdo a la edad que presente un individuo.

Por otro lado, aunque las diferencias atribuibles a sexo y edad encontradas en este estudio resultaron significativas, éstas fueron menos marcadas y se encontraron en puntos distintos a las encontradas por Suazo et al. en cadáveres de individuos españoles, lo que puede estar relacionado la técnica de obtención de las medidas de grosor tisular.
No se apreciaron diferencias significativas entre individuos de un mismo sexo y grupo etáreo, pudiéndose inferir que existe una homogeneidad morfológica en el grosor tisular de nuestra población, según sexo y grupo etáreo.

La ciencia forense ha avanzado considerablemente en los últimos años, a la par con los avances y progresos tecnológicos, haciendo de estas técnicas un método cada vez más exacto y eficaz en el proceso de identificación médico-legal.

El uso de ultrasonografía para determinar el grosor de los tejidos blandos, puede ser de gran ayuda, ya que es un método económico, rápido, no invasivo que entrega, además, una excelente imagen, siendo su principal limitante la necesidad de un operador entrenado. 
SUAZO, G. I. C.; SALGADO, A. G. E.; CANTíN, L. M. G. E valuación ultrasonográfica del tejido blando facial en adultos chilenos. Int. J. Morphol., 25(3):643-648, 2007.

SUMMARY; Varied methods exist to realize the medicolegal identification in cadaveric remains or skeletons, one of them is the identification for forensic sculpture. For this certain references are needed in the relation between osseous and soft tissue. The objective of this investigation was to determine face tissue thicknesses by means of ultrasonography and to correlate them with age and sex. There was selected a sample of 48 persons, between 21 and 50 years of age of both sexes, which they consulted to the service of imagenología of Talca's Multimedical Clinic distributed in two groups: A group with 12 men and 12 women between 21 and 35 years of age; B group with 12 men and 12 women between 36 and 50 years of age. For this study an ecographe was used, General Electric $\AA$, model voluson 730 expert, to which the tissue thicknesses measured up in 15 medium and paramedium craniofacial points, which were correlated by the parameters of sex and age. The results of the measurement of the tissue thicknesses showed significant differences between age groups and between sexes. Significant differences were not observed, between the persons of the same sex of the same age group.

KEY WORDS: Forensic; Facial reconstitution; Soft-tissue thicknesses; Identification.

\section{REFERENCIAS BIBLIOGRÁFICAS}

Bergman, R.T. Cephalometric soft tissue facial analysis. Am. J. Orthod. Dentofacial. Orthop., 116(4):373-89, 1999.

Claes, P.; Vandermeulen, D.; De Greef, S.; Willems, G. \& Suetens, P. Craniofacial reconstruction using a combined statistical model of face shape and soft tissue depths: methodology and validation. Forensic Sci. Int., 159(1):147-58, 2006.

De Greef, S. \& Willems, G. Three-dimensional cranio-facial reconstruction in forensic identification: latest progress and new tendencies in the $21^{\text {st }}$ century. $J$. Forensic Sci., 50(1):472-7, 2005.

De Greef, S.; Vandermeulen, D.; Mollemans, W.; Suetens, P. \& Willems, G. Large-scale in-vivo Caucasian facial soft tissue thickness database for craniofacial reconstruction. Forensic Int. Sci.; 159(1):126-146, 2006.

Domaracki, M. \& Stephan, C.N. Facial soft tissue thicknesses in Australian adult cadavers. J. Forensic Sci., 51(1):5-10, 2006.

El-Mehallawi I. H. \& Soliman, E. M. Ultrasonic assessment of facial soft tissue thicknesses in adult Egyptians. Forensic Int. Sci., 117(1-2): 99-107, 2001.

Smith, S.L. \& Buschang, P. H. Midsagittal facial tissue thicknesses of children and adolescents from the Montreal growth study. J. Forensic Sci., 46(6):1294$302,2001$.
Stephan, C. N. \& Henneberg, M. Building faces from dry skulls: are they recognized above chance rates?. $J$. Forensic Sci., 46(3):432-40, 2001.

Stephan, C.N.; Norris, R.M. \& Henneberg, M. Does sexual dimorphism in facial soft tissue depths justify sex distinction in craniofacial identification? J. Forensic Sci., 50(3):513-8, 2005.

Suazo, G. I. C.; Pérez, R. F. J. \& Torres, M. S. R. Grosores titulares faciales en cadáveres de españoles y su aplicación en la identificación médicolegal. Int. J. Morphol., 25(1):109-16, 2007.

Teke, A. Medicina legal y criminalística. Ediciones Jurídicas de Santiago, Santiago de Chile, 2004. pp.193202, 223-4.

Utsuno, H.; Kageyama, T.; Deguchi, T.; Umemura, Y.; Yoshino, M.; Nakamura, H. et al. Facial soft tissue thickness in skeletal type I Japanese children. Forensic Sci. Int., doi:10.1016/j.forsciint.2007.01.004, 2007.

Utsuno, H.; Kageyama, T.; Deguchi, T.; Yoshino, M.; Miyazawa, H. \& Inoue, K. Facial soft tissue thickness in Japanese female children. Forensic Sci. Int., 152(23):101-7, 2005

Vandermeulen, D.; Claes, P.; Loeckx, D.; De Greef, S.; Willems, G. \& Suetens, P. Computerized craniofacial reconstruction using CT-derived implicit surface representations. Forensic Sci. Int., 159(1),164-74, 2006. 
SUAZO, G. I. C.; SALGADO, A. G. E.; CANTíN, L. M. G. Evaluación ultrasonográfica del tejido blando facial en adultos chilenos. Int. J. Morphol., 25(3):643-648, 2007.

Wilkinson, C. M. In vivo facial tissue depth measurements for white British children. J. Forensic Sci., 47(3): 459$65,2002$.

Wilkinson, C. M.; Motwani, M. \& Chiang, E. The relationship between the soft tissues and the skeletal detail of the mouth. J. Forensic Sci., 48(4):729-32, 2003.
Dirección para correspondencia:

Dr. Iván Suazo Galdames

Unidad de Anatomía Normal

Universidad de Talca

Avenida Lircay s/n oficina $\mathrm{N}^{\circ} 104$

Talca - CHILE

Fono 56-71-201682

Email: isuazo@utalca.cl

Recibido : 20-06-2007

Aceptado: 20-07-2007 